\title{
Current Status and Technological Limitations of Hybrid Superconducting-normal Single Electron Transistors
}

\author{
Giampiero Amato and Emanuele Enrico \\ The Quantum Research Laboratory, INRIM, Turin
}

Italy

\section{Introduction}

Since the original paper from Josephson on tunnel phenomena occurring in superconducting junctions (Josephson, 1962), superconductors have been widely studied by metrologists, because of the quantistic origin of most effects observed in such class of materials. There is, in fact, an intimate relationship between the definition of more accurate and stable standards and Quantum Mechanics. Indeed, the Josephson Voltage Standard (JVS) is believed to be a fundamental quantum physical effect, which is the same everywhere, and at all times.

Tunnel effect has, however, several other implications, one of them being the possibility of localizing a single electron in space. An electric current can flow through the conductor because some electrons are free to move through the lattice of atomic nuclei. The charge transferred through the conductor determines the current. This transferred charge can have practically any value, in particular, a fractional charge value as a consequence of the displacement of the electron cloud against the lattice of atoms. This shift can be changed continuously and thus the transferred charge is a continuous quantity, not quantized at all! If a discontinuity in space is introduced, e.g. by means of a tunnel junction, electric charge will move through the system by both continuous and discrete processes. Since, from a semi-classical point of view, only discrete electrons can tunnel through junctions, charge will accumulate at the surface of the electrode against the isolating layer, until a high enough bias has built up across the tunnel junction, and one electron will be transferred. This argument, which will be substantiated in a purely quantistic view in the following, led K. Likharev (Likharev, 1988) to coin the term 'dripping tap' as an analogy of this process. In other words, if a constant current I is forced to pass through a single tunnel junction, the so called Coulomb oscillations will appear with frequency $\mathrm{f}=\mathrm{I} / \mathrm{e}$ where e is the charge of an electron. The current biased tunnel junction is a very simple circuit able to show the controlled transfer of electrons.

Differently from the JVS, devices capable to control the electron transfer one-by-one are still far to reach the accuracy level necessary for metrological applications. Controlling and counting electrons one-by-one in an electrical circuit will give the possibility of realizing a quantum standard for electrical current. It is important to remember that in the SI system, the base electrical unit is the ampere, but, nowadays, the primary electrical standards are the 
quantum Hall effect (QHE) resistance standard and the JVS. Both are believed to be fundamental physical effects and widely used in metrological laboratories. The quantum Hall resistance $\mathrm{R}$ and Josephson voltage $\mathrm{V}$ are given by:

$$
\begin{array}{ll}
\mathrm{R}=\mathrm{R}_{\mathrm{k}} / \mathrm{i} & \left(\mathrm{R}_{\mathrm{k}}=\mathrm{h} / \mathrm{e}^{2}\right) \\
\mathrm{V}=\mathrm{nf} / \mathrm{K}_{\mathrm{j},} & \left(\mathrm{K}_{\mathrm{j}}=2 \mathrm{e} / \mathrm{h}\right)
\end{array}
$$

where $\mathrm{i}$ and $\mathrm{n}$ are integers, $\mathrm{f}$ is a frequency, $\mathrm{h}$ and e are fundamental constants, namely, the Planck's constant and the electron charge.

The QHE ohm and Josephson volt are linked to the ampere via difficult experiments, with a relatively high uncertainty (Flowers, 2004). In consequence, the QHE and JVS are referred to as 'representations' of the SI ohm and volt. To address this inconsistency, the International Committee of Weights and Measures (CIPM) recommended the study of proposals to redefine some of the SI units in 2011.

A quantum electrical standard, based on single electron transport, yields a current given by:

$$
\mathrm{I}=\mathrm{n}^{\prime} \mathrm{f}^{\prime} \mathrm{e}
$$

where the current I through the transistor is defined by the number $\mathrm{n}^{\prime}$ of elementary charges (e) injected in one period and $\mathrm{f}^{\prime}$ is the frequency.

There are two basic requirements for a transistor to act as an electron turnstile. The first is that the charging energy for an electron confined into an island of material in between two tunnel junction must be larger than the thermal energy of electrons. This condition can be written as $\mathrm{e}^{2} / 2 \mathrm{C}_{\Sigma}>>\mathrm{kT}$, where $\mathrm{C}_{\Sigma}$ is the total capacitance of the device. This first condition has two direct technological and physical consequences: to observe Coulomb blockade, junctions with lateral dimension in the 10-100 $\mathrm{nm}$ range are required so to have $\mathrm{C}_{\Sigma} \leq 10^{-16} \mathrm{~F}$. Of course, the measurement must be carried out at cryogenic temperatures, with typical values in the $\mathrm{mK}$ range. What is important to underline here is the need of nano-technologies to realize the device. Standard photolithograpy, widely employed by microelectronic industries for high density package of devices in a single chip, can hardly approach the geometrical limit required, so, Electron Beam Lithography (EBL) is commonly used for the purpose.

The second condition to be fulfilled by an electron turnstile is more related to the basics of Quantum Mechanics. In a classical picture it is clear if an electron is either on an island or not. In other words, the localization is implicitly assumed in a classical formalism. However, in a more precise quantum mechanical description, the number of electrons $\mathrm{N}$ localized on an island are in terms of an average value $\langle\mathrm{N}\rangle$ which is not necessarily an integer. The so-called Coulomb blockade effect prevents island charging with an extra electron, that is $|N-\langle N\rangle|^{2}<<1$. Clearly, if the tunnel barriers are not present, or are fairly opaque, no island charging or electrons localization on a quantum dot will be accomplished, because of the absence of confinement for an electron within a certain volume. From a quanto-mechanical point of view, the condition $|\mathrm{N}-\langle\mathrm{N}\rangle|^{2<<1}$ requires for the time $\mathrm{t}$ which an electron resides on the island, $\mathrm{t}>\Delta \mathrm{t} \geq \mathrm{h} / \Delta \mathrm{E}$. Let us assume that for moderate bias and temperature at most one extra electron resides on the island at any time, so the current cannot exceed $\mathrm{e} / \mathrm{t}$. This means that the energy uncertainty on the electron must be $\Delta \mathrm{E}<\mathrm{V}_{\mathrm{b}}$, where $\mathrm{V}_{\mathrm{b}}$ is the applied bias. Trivial calculations lead to the conclusion that the resistance of the tunnel junctions $R_{T}=V_{b} / I>>h / e^{2}$. The last quantity is the von Klitzing constant $R_{K}$, known to be $R_{K} \equiv 25813$ $\Omega$. More rigorous theoretical studies on this issue have supported this conclusion (Zwerger 
\& Scharpf, 1991). Experimental tests have also shown this to be a necessary condition for observing single-electron charging effects (Geerligs et al., 1989).

An important experiment, in which all the three electrical standards are joined together, is the Metrological Triangle. We can describe this experiment like a sort of quantum validation of the Ohm's Law. Joining eqs. (1), (2) and (3), we will yield the product $R_{k} K_{j} e$. This is expected to be exactly 2. Any discrepancy from this value will indicate a flaw in our understanding of one or more of these quantum effects. This experiment will be an important input into the CIPM deliberations on the future of the SI. It is one of the higher priorities in fundamental metrology today.

Current pumps based on mesoscopic metallic tunnel junctions have been proposed in the past (Geerligs et al., 1990; Pothier et al., 1992) and demonstrated to drive a current with a very low uncertainty (Keller et al., 1996). Unfortunately, these systems are difficult to control and relatively slow (Zimmerman \& Keller, 2003). Amongst the various attempts to overcome these limitations by using e.g. surface-acoustic-wave driven one-dimensional channels (Talyanskii et al. 1997), superconducting devices (Vartiainen et al., 2007; Niskanen et al., 2003; Lotkhov, 2004; Governale et al., 2005; Kopnin et al. ,2006; Mooij and Nazarov, 2006, Cholascinski \& Chhajlany, 2007) and semiconducting quantum dots (Blumenthal et al., 2007), a system based on hybrid superconducting-metal assemblies and capable of higher accuracy has been recently proposed (Pekola et al., 2008).

\section{Theorethical background}

\subsection{The Orthodox theory}

In the present chapter, we will review the Orthodox (Averin \& Likharev, 1991) theory for the Normal-metal Single Electron Transistor (n-SET) with the aim of extending it to the case of hybrid Superconductor/Normal structures. This model, which will be discussed in a following section, enables to predict the h-SET performances when different superconductors are employed.

For clarity purposes, we will give a heuristic treatment for the n-SET but without any lack of generality, while a more detailed discussion will be devoted to the hybrid case.

The energy that determines the transport of electrons through a single-electron device is Helmholtz's free energy which is defined as difference between total energy $E_{\Sigma}$ stored in the device and work done by power sources. The total energy stored includes all the before mentioned energy components that have to be considered when charging an island with an electron. The change in Helmholtz's free energy a tunnel event causes is a measure of the probability of this tunnel event. The general fact that physical systems tend to occupy lower energy states, is apparent in electrons favoring those tunnel events which reduce the free energy.

In the framework of the Orthodox theory (Averin \& Likharev, 1991) the tunneling rate $\Gamma$ across a single junction between two normal metal electrodes can be extracted using the Golden Rule as:

$$
\begin{aligned}
& \Gamma^{+}=e^{2} R_{T}^{-1} \int_{-\infty}^{+\infty} f(E, T)[1-f(E-\Delta F, T)] d E \\
& \Gamma^{-}=e^{2} R_{T}^{-1} \int_{-\infty}^{+\infty} f(E+\Delta F, T)[1-f(E, T)] d E
\end{aligned}
$$


where $\Delta \mathrm{F}$ is the variation in the Helmholtz free energy of the system. Integration of (4.1) yields:

$$
\Gamma=-\Delta F e^{2} R_{T}^{-1}\left[1-\exp \left(\Delta F / k_{B} T\right)\right]
$$

It can be easily concluded that, in the low $\mathrm{T}$ limit, $\Gamma=0$ when $\Delta \mathrm{F}>0$, whereas:

$$
\Gamma=-\Delta F e^{2} R_{T}^{-1} \quad \Delta \mathrm{F}<0
$$

The quantity $\Delta \mathrm{F}$ for a n-SET with i junctions can be written in the following way:

$$
\Delta F_{i}^{ \pm}=e\left(e / 2 C_{\Sigma} \pm V_{i}\right)
$$

where $i=1,2$ in a single-island $n-S E T, V_{i}$ is the voltage bias across the junctions. Here, we are dealing with 4 different equations, which consider the possibility for one electron to enter in $(+)$ or to exit from (-) the island both from junctions 1 or 2 .

Eq. (5) gives a perspicuous representation of the Helmoltz free energy for an island limited by two tunnel junctions. The energy $\mathrm{E}_{\mathrm{c}}=\mathrm{e}^{2} / 2 \mathrm{C}_{\Sigma}$ is clearly the energy stored in the device, whereas $\pm \mathrm{eVi}$ represents the work done by the power sources.

\subsection{The Normal-Insulator-Normal SET}

In Fig. 1 a SET equivalent circuit is displayed. First, it is helping to write the equations for a double junction system, and then to correct them when a gate contact is added.

The charge $\mathrm{q}_{\mathrm{i}}$ at the $\mathrm{i}$-th junction can be written as $\mathrm{q}_{\mathrm{i}}=\mathrm{C}_{\mathrm{i}} \mathrm{V}_{\mathrm{i}}$, so, the total charge into the island is $q=q_{2}-q_{1}+q_{0}=-n e+q_{0}$ where $q_{0}$ is the background charge inside the island and $n=n_{1}-n_{2}$ is an integer number indicating the electrons in excess.

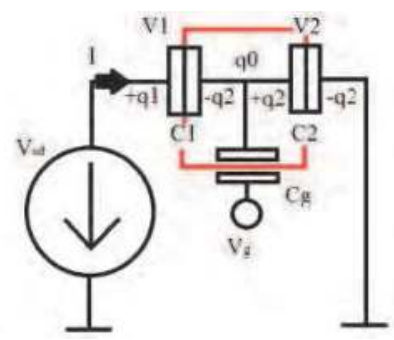

Fig. 1. Equivalent circuit of a single-island, two-junctions SET

The voltage bias across the $\mathrm{i}$-th junction is then:

$$
V_{i}=\left[C_{(3-i)} V_{S D}+(-1)^{i}\left(q_{0}-n e\right)\right] C_{\Sigma}^{-1}
$$

where $V_{S D}$ is the bias across the device $\left(V_{S D}=\Sigma V_{i}\right)$ and $C_{\Sigma}=\Sigma C_{i}$.

To add the contribution of the gate contact in the device, we can simply take into account for effect of the gate electrode on the background charge $\mathrm{q}_{0}$. This quantity can be changed at will, because the gate additionally polarizes the island, so that the island charge becomes: 


$$
q=-n e+q_{0}+C_{g}\left(V_{g}-V_{2}\right)
$$

with $\mathrm{V}_{\mathrm{g}}$ the gate voltage.

Now, after, some trivial calculations, one can write the final relationship giving the voltage bias across the i-th junction in Single Electron Transistor (SET) composed by 1 island surrounded by 2 tunnel junctions:

$$
V_{i}=C_{\Sigma}^{-1}\left\{\left[C_{(3-i)}+C_{g} \delta_{i, 1}\right] V_{S D}+(-1)^{i} C_{g} V_{g}+(-1)^{i+1} n e+q_{0}\right\}
$$

where $\delta_{i, 1}$ is the Dirac's function and $C_{\Sigma}=\Sigma C_{i}+C_{g}$.

By combining (8) and (5) it is possible to explicitly write the equations governing the free energy change in a system with two tunnel junctions and a gate electrode. For example, under the particular conditions: $\mathrm{q}_{0}=0, \mathrm{R}_{1}=\mathrm{R}_{2}$ and $\mathrm{C}_{1}=\mathrm{C}_{2}=\mathrm{C}>>\mathrm{C}_{\mathrm{g}}$, one gets:

$$
\begin{aligned}
& \Delta F_{1}^{ \pm}=2 E_{c}\left( \pm n \mp n_{g}+1 / 2\right) \pm V_{S D} / 2 \\
& \Delta F_{2}^{ \pm}=2 E_{c}\left(\mp n \pm n_{g}+1 / 2\right) \pm V_{S D} / 2
\end{aligned}
$$

where $\mathrm{n}_{\mathrm{g}}=\mathrm{C}_{\mathrm{g}}\left(\mathrm{V}_{\mathrm{g}}-\mathrm{V}_{2}\right)$ and $\mathrm{E}_{\mathrm{c}}=\mathrm{e}^{2} / 2 \mathrm{C}$.

In order to model the behavior of such a complex system, some simplifying assumptions are needed. First of all, we consider the tunneling events as instantaneous and uncorrelated, say, one is occurring at a time. Since any single-electron tunneling event changes the charge state of the island, at least two states are required for current transport.

Having the rates of tunneling through the two junctions at hand we can now define the rates of elementary charge variation for the island as:

$$
\Gamma_{n+1, n}=\bar{\Gamma}_{1}(n)+\vec{\Gamma}_{2}(n) \quad \Gamma_{n-1, n}=\bar{\Gamma}_{2}(n)+\vec{\Gamma}_{1}(n)
$$

With the aid of the above considerations it is possible to define a master equation that governs the behavior of the system, whose solution is (Ingold \& Nazarov, 1992):

$$
\Gamma_{n, n+1} P_{n+1}=\Gamma_{n+1, n} P_{n}
$$

where $P_{n}$ is the probability distribution for the island charge state.

Taking as a starting state that one with no excess charge in the island and considering that only the nearest neighbors states are connected by non-null rates, the probability distribution can be derived from eq. (11) as:

$$
\begin{array}{ll}
P_{n}=P_{0} \prod_{m=0}^{n-1} \Gamma_{m+1, m} / \Gamma_{m, m+1} & \mathrm{n}>0 \\
P_{n}=P_{0} \prod_{m=n+1}^{0} \Gamma_{m-1, m} / \Gamma_{m, m-1} & \mathrm{n}<0
\end{array}
$$

where the free parameter $P_{0}$ can be extracted from the normalization condition $\sum_{-\infty}^{+\infty} P_{n}=1$. Being the steady-state currents through the two junctions equal to I we can write: 


$$
I=e \sum_{-\infty}^{+\infty} P_{n}\left[\overrightarrow{\Gamma_{1}}(n)-\overleftarrow{\Gamma_{1}}(n)\right]=e \sum_{-\infty}^{+\infty} P_{n}\left[\overrightarrow{\Gamma_{2}}(n)-\overleftarrow{\Gamma_{2}}(n)\right]
$$

It's trivial to note that in the $\mathrm{T}=0$ limit the terms $\left(\overrightarrow{\Gamma_{1}}-\overline{\Gamma_{1}}\right.$ and $\left.\overrightarrow{\Gamma_{2}}-\overline{\Gamma_{2}}\right)$ of eq. (13) are identically null for some values of $\mathrm{V}_{\mathrm{SD}}$ and $\mathrm{n}_{\mathrm{g}}$. In these states it is also noted that the probability distribution $\mathrm{P}_{\mathrm{n}}=1$ for a well defined value of $\mathrm{n}$. This means that these regions are stable in terms of the number of charges on the island and both tunnel junctions are in the so-called Coulomb Blockade state.

In the zero temperature limit, by imposing $\Delta F_{i}^{ \pm}=0$, one is able to write down the equations providing the dependence of $\mathrm{V}_{\mathrm{SD}}$ on $\mathrm{n}_{\mathrm{g}}$ at the boundaries between the regions in which tunneling is allowed $\left(\Delta F_{i}^{ \pm}<0\right)$ and forbidden $\left(\Delta F_{i}^{ \pm}>0\right)$. Without going into details on this rather simple calculation, we can easily observe that such dependence is linear, with slopes given by $C_{g} /\left[C_{(3-i)}+C_{g} \delta_{i, 1}\right]$ and intercepts related to the number $\mathrm{n}$ of excess electrons into the island. These lines give rise to the well-known Stability Diagram for a n-SET depicted in Fig. 2.

Diamonds in the Stability Diagram are representative for the region where tunneling is inhibited $\left(\Delta F_{i}^{ \pm}>0\right)$. They are defined by two families of parallel lines having positive (1st junction) and negative (2nd junction) slopes, respectively. Outside such regions, current can flow freely across the device, whereas the control of the charging state at single-electron level can be obtained only when the working point with coordinates $n_{g}, V_{S D}$ lies inside a stable diamond.

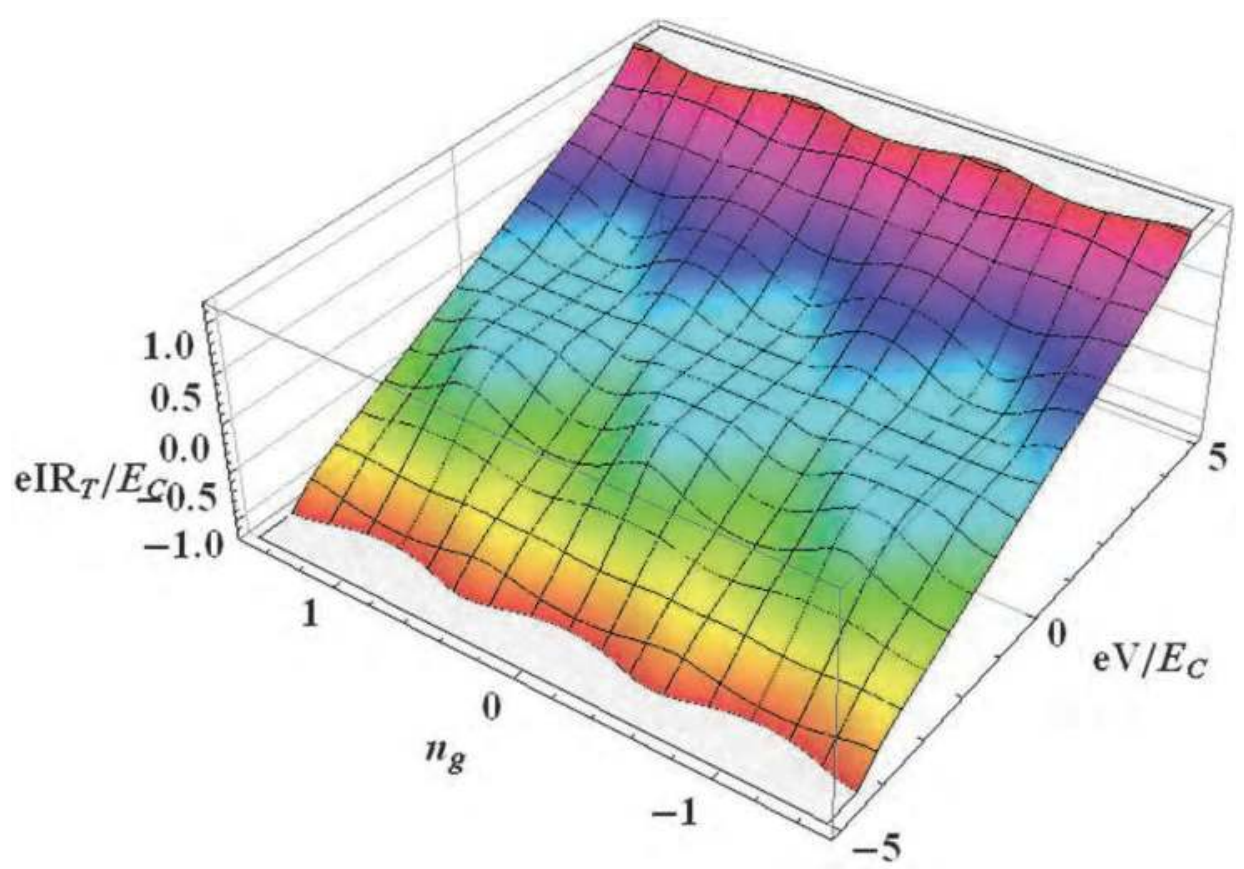

Fig. 2. Stability Diagram for a n-SET 
It is important to stress that the stable states in the case of n-SETs have a single degeneracy point in which the the states with $n$ or $n+1$ are equiprobable (Fig. 2).

The only location on the stability diagram, and therefore the only set of coordinates $n_{g}, V_{S D}$ which allows the system to switch from one stable state to another passes through the degeneracy point where the bias voltage $V_{S D}$ is nil in any circuit configuration. Then, the reader can understand how a simple n-SET can control the number of elementary charges in excess on the island, solely, but not the flow of single electrons from source to drain electrodes. This because the system switch from $n$ to $n+1$ can occur either through the forward tunneling in the first junction or the backward tunneling in the second junction, with the same probability. In other words, $V_{S D}=0$ implies that no directionality for the events is defined, that is, the n-SET cannot work as a turnstile.

For $\mathrm{V}_{\mathrm{SD}} \neq 0$, the current can freely flow across the device in well-defined $\mathrm{V}_{\mathrm{g}}$ intervals. The so-called SET oscillations can then be observed (Fig. 3).

Investigators have tried to circumvent this problem by using multi-island electron pumps (Zimmerman \& Keller, 2003). In such devices some islands are in series and driven by their own gate contact. Sinusoidal waveforms for each of these gates are shifted in phase, so to ensure that successive tunnel process occur from the first to the last junction. The relatively complicated experiment with such a slow device yields an output value for the singular current much lower than the limit $\left(10^{-10-10^{-9}} \mathrm{~A}\right)$ necessary for carrying out the Metrological Triangle experiment with the required accuracy.

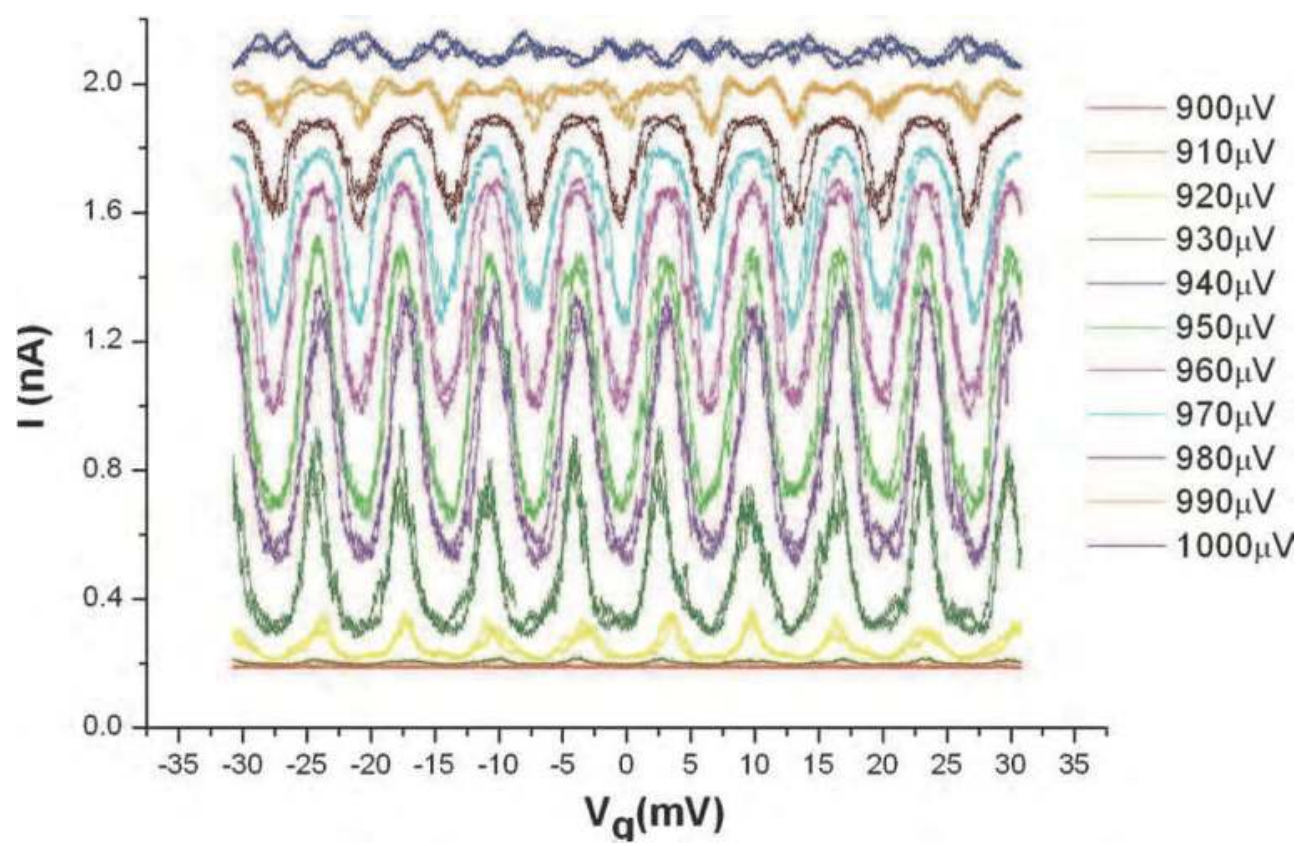

Fig. 3 The SET oscillations occurring when $V_{S D} \neq 0$. Values for $V_{S D}$ are given on the right side of the Fig. With scanning $\mathrm{V}_{\mathrm{g}}$, we find peaks representing the current flow through the device, when $\Delta F_{i}^{ \pm}<0$ (outside of the diamonds in Fig. 2), and minima related to $\Delta F_{i}^{ \pm}>0$. 


\subsection{The hybrid SET}

Hybrid superconducting-metal assemblies have been recently proposed and shown to be capable of higher accuracy (Pekola et al., 2008). From a technological point of view, this assembly is composed by a normal-metal island sandwiched by two superconducting electrodes (SNS), or the reverse (NSN) scheme. For the purpose of this chapter, the theoretical description is the same for both the arrangements.

In the following chapter, eqs. (4) will be rewritten in the case of NIS junction and applied to h-SET.

\subsubsection{Tunneling in a S-I-N junction}

Typical applications of SIN junctions are microcoolers (Nahum et al., 1994; Clark et al., 2005; Giazotto et al., 2006) and thermometers (Nahum \& Martinis, 2003; Schmidt et al. 2003; Meschke et al. 2006; Giazotto et al., 2006). In these applications, SIN junctions are usually employed in the double-junction (SINIS) geometry. The opposite NISIN geometry has gathered less attention. Recently, there has been interest in SINIS structures with considerable charging energy. They have been proposed for single-electron cooler applications (Pekola et al., 2007; Saira et al., 2007) that are closely related to the quantized current application (Pekola et al., 2008). Thermometry in the Coulomb-blocked case has been considered, too (Koppinen et al., 2009).

In the case where the superconductor in study is well below its transition temperature $\left(\mathrm{T}_{\mathrm{S}}<\mathrm{T}_{\mathrm{c}}\right)$ it can be assumed for the superconducting gap $\Delta$ that $\Delta\left(\mathrm{T}_{\mathrm{S}}\right)=\Delta(0)=\Delta$.

Because the number of particles for a given amount of energy must be the same in the superconducting state (quasi-particles) and in the normal one (free electrons), the relationship:

$$
g_{s}(E) d E=g_{n}(\varepsilon) d \varepsilon
$$

must hold, and then:

$$
g_{s}(E)=g_{n}(\varepsilon) E\left(E^{2}-\Delta^{2}\right)^{-1 / 2} \theta(E-\Delta)
$$

where $\theta$ is the Heaviside's step function.

Then, the density of states in a superconductor can be written as:

$$
g_{s}(E) \cong g_{n}(0) E\left(E^{2}-\Delta^{2}\right)^{-1 / 2} \theta(E-\Delta)
$$

by considering that:

1. all the energy terms at low temperatures have significant values of the order of $k_{B} T$ (which is several orders of magnitude less than the Fermi energy, $\mathrm{k}_{\mathrm{B}} \mathrm{T}<<\mathrm{E}_{\mathrm{F}}$ );

2. the energies are measured with respect to the Fermi level $\left(\varepsilon=0\right.$ at $\left.\mathrm{E}_{\mathrm{F}}\right)$;

3. $g_{n}(\varepsilon) \approx g_{n}(0)$.

A further assumption is that the electrons in the metal and the quasiparticles in superconductor are weakly interacting and at thermal equilibrium due to the high potential barrier of the dielectric layer. It is then possible, to consider tunneling as a perturbation and to apply the Golden Rule approach. The dominating current transport mechanism in a NIS junction is single-electron tunneling between the normal metal and the quasi-particle states of the superconductor. 
The equations governing the rate of tunneling back and forth in a NIS can be written in a similar way to that for the NIN system by simply adding a term proportional to the superconductor density of states:

$$
\begin{aligned}
& \Gamma^{+}=e^{2} R_{T}^{-1} \int_{-\infty}^{+\infty} n_{S}(E) f\left(E, T_{S}\right)\left[1-f\left(E-\Delta F, T_{N}\right)\right] d E \\
& \Gamma^{-}=e^{2} R_{T}^{-1} \int_{-\infty}^{+\infty} n_{S}(E) f\left(E+\Delta F, T_{N}\right)\left[1-f\left(E, T_{S}\right)\right] d E
\end{aligned}
$$

where $T_{s}$ and $T_{N}$ are temperatures for the superconductor and normal electrodes, respectively and $n_{s}=g_{s}(E) / g_{N}(0)$.

For $\mathrm{T}=0$, the corresponding of eq. (4.3) is found for the SIN case:

$$
\Gamma=e^{-2} R_{T}^{-1} \sqrt{\Delta F^{2}-\Delta^{2}} \quad \Delta F \leq-\Delta
$$

whereas $\Gamma=0$ for $\Delta \mathrm{F}>-\Delta$ (the other two solutions cannot be considered because transitions are allowed only for negative free energy variations).

Finding a solution for eq. (17) is not a trivial task and will not be reported here, but some words are deserved to the tunneling effects occurring in the SIN junction at voltages values below gap. Here, if the condition $\mathrm{K}_{\mathrm{b}} \mathrm{T}_{\mathrm{N}}<<\Delta \mathrm{F}$ (i.e., if $\mathrm{T}_{\mathrm{N}}<<1.76 \mathrm{~T}_{\mathrm{c}}$ ) is fulfilled, the rate of tunneling through the SIN junction is given by:

$$
\Gamma_{T}\left(V, T_{N}\right)=\Gamma_{0} \exp \left[(\Delta F-\Delta) / k_{b} T_{N}\right]
$$

the quantity $\Gamma_{0}=\Delta e^{-2} R_{T}^{-1} \sqrt{\pi k_{b} T_{N} / 2 \Delta}$ being called the characteristic rate and approximately representing the tunneling rate when the free energy variation approaches the gap.

From eq. (19) it can be seen that for free energy variations below the gap the tunneling rate strongly depends on temperature. This opens the possibility of using this type of junction as a thermometer at low temperature. As a drawback, limits in the accuracy of electron counting for metrological applications of h-SETs can arise, as discussed in the next chapters.

\subsubsection{Stability diagram for $h-S E T$}

Following the same n-SET master equation approach for the SINIS system, it is now possible to combine eqs. (9) and (17) in order to consider the case in which the mesoscopic tunnel junctions charging energy is not negligible and the central island is coupled to a gate electrode.

Results from calculations of the electrical characteristics for the previous ideal system are shown in Fig. 4. Using a similar procedure for the n-SET device we can study the h-SET behavior at temperature $\mathrm{T}->0 \mathrm{~K}$ in order to extract the modified stability diagram.

It is observed from eq. (18) that when $\Delta \mathrm{F}>-\Delta$, the tunneling rate is nil (in principle) and the junction does not allow for the electron flow. The areas in the stability diagram in which such conditions hold identify the stable regions with a defined number of elementary charges on the island (n). 


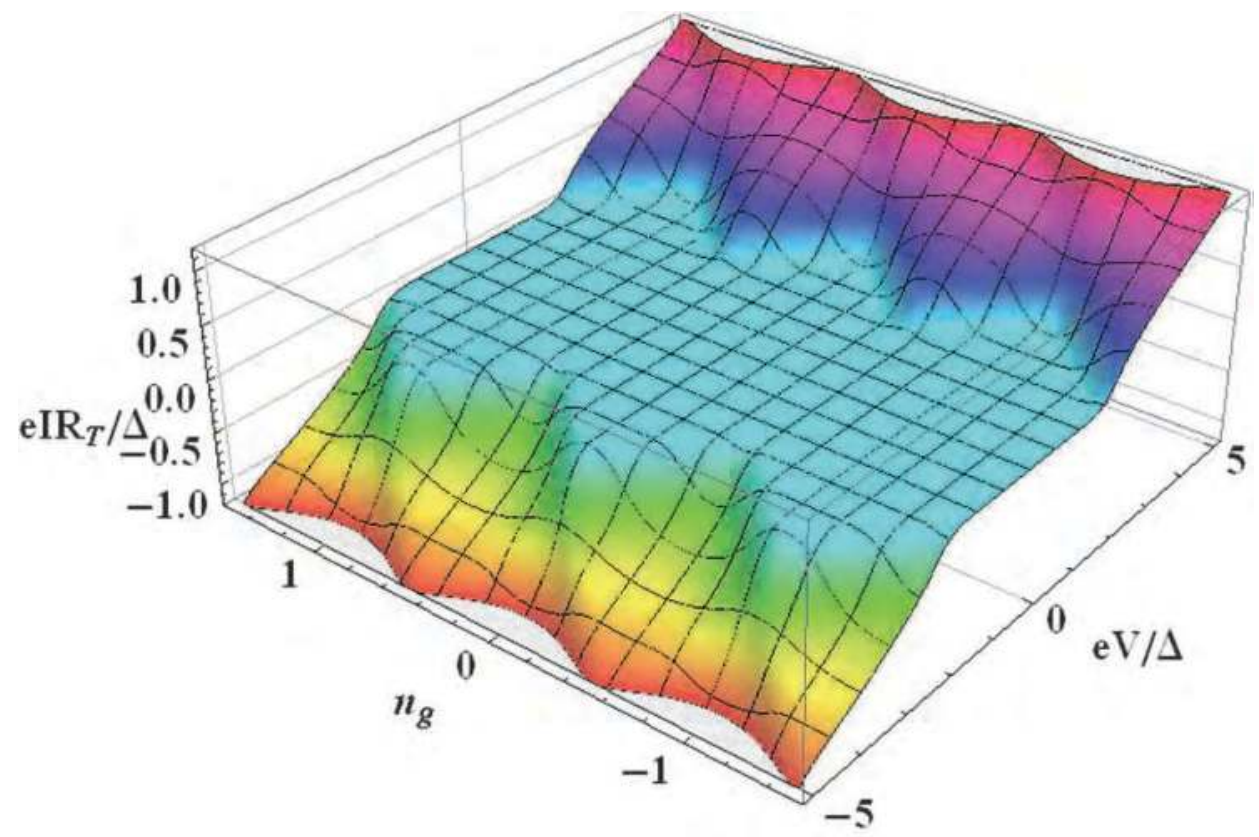

Fig. 4. 3-dimensional view of the Stability Diagram for a h-SET

The present formalism allows us to treat the hybrid assembly in the same way as the normal system. Then, very trivial calculations let us to extract the equations for the two families of straight lines defining the boundaries between regions of allowed and forbidden tunneling, with an offset value with respect to the lines in the normal case of $2 \Delta$ (Fig. 5).

The dashed lines in Fig. 5 define the regions of inhibited tunneling, as in the case of the n-SET (purple areas). In the hybrid system, each of them is shifted by an offset $2 \Delta$, defining the blue regions where the mechanism of tunnel inhibition is the band offset at the SIN junction.

When the Current across a Single Electron Tunneling device is recorded as a function of the $V_{S D}$, for different $n_{g}$ values, a family of Current-Voltage characteristics is obtained. This means we are moving along parallel vertical pathways on the Stability Diagram. The extension of the Coulomb gap, obviously depends on the $n_{g}$ value: in a Normal SET it periodically oscillates from 0 to e/2C, with a periodicity of one unit (see Fig. 6).

It is then interesting to compare the Current-Voltage characteristics of the Normal and Hybrid SETs. This comparison, reported in Fig. 6, clearly indicates a broadening of the conduction gap in the hybrid structure. The gap oscillates with tuning $\mathrm{V}_{\mathrm{g}}$ from $\Delta$ to $\Delta+\mathrm{e} / 2 \mathrm{C}$. In a few words, the presence of the superconducting gap broadens the region of inhibited tunneling, whose width never equals to zero.

In this configuration for the h-SET, the degeneracy point linking the stable states is suppressed by a region in the $V_{S D}-n_{g}$ space where the pathway from point $A$ to point $B$ occurs with negligible backward tunneling at both junctions $\left(\mathrm{V}_{\mathrm{SD}}>0\right)$ and without departing from the stable regions. E.g., with $\mathrm{V}_{\mathrm{g}}$ oscillating between the states $\mathrm{A}$ and $\mathrm{B}$, one can move a single electron per cycle from source electrode to drain with a well defined directionality given by the sign of $\mathrm{V}_{\mathrm{SD}}$. 


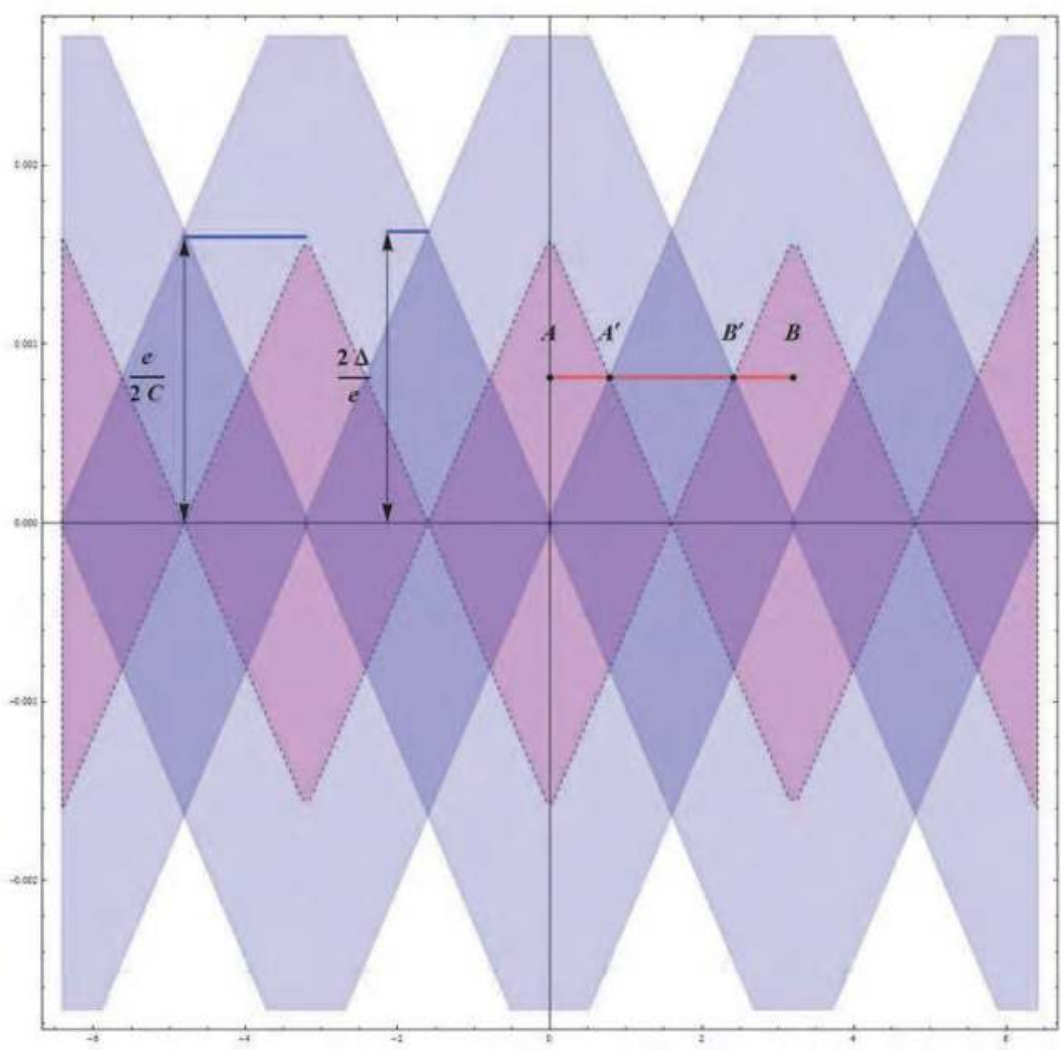

Fig. 5. Plan view of the Stability Diagram for a h-SET. For clarity purposes, $\Delta$ is given in eV, so to compare directly with $\mathrm{V}_{\mathrm{SD}}$.

In the next section we will analyze some of the possible effects that can alter the process of controlled transport of electric charges in a h-SET turnstile configuration.

The extension of the Coulomb blocked region to $V_{S D}$ values $\neq 0$ is the peculiar feature of the hybrid assembly. This opens the possibility for such a device to operate as a turnstile. In fact, we can operate the device along the pathway between points $A$ and $B$ with $V_{S D} \neq 0$ (Fig. 5). From points $\mathrm{A}$ and $\mathrm{A}^{\prime}$ ( $\mathrm{B}^{\prime}$ and $\mathrm{B}$ ) tunneling inhibition is accomplished thanks to the Coulomb Energy $e^{2} / 2 C(\Delta F<0)$, whereas in the intermediate region $A^{\prime} B^{\prime}$, the presence of the superconducting gap is the limiting mechanism $(0<\Delta \mathrm{F}<\Delta)$.

\subsubsection{Error sources in hybrid SET}

The following treatment on the error sources in Hybrid SET will not be exhaustive, since second-order (e.g. co-tunneling), and technology-related (e.g. Adreev's reflections at the oxide pinholes) effects, will not be discussed. We will focus on a sort of "ideal" h-SET, in order to determine the optimal conditions for turnstile operation. 
It is rather intuitive that small $\mathrm{V}_{\mathrm{SD}}$ values lead to an increased probability for tunneling events in the backward direction, according to the relationship (Pekola et al., 2008):

$$
\Gamma_{b} \propto \exp \left(-e V_{S D} / k_{B} T_{N}\right)
$$

where $\Gamma_{b}$ is the rate of backward tunneling $k_{B}$ the Boltzmann constant and $T_{N}$ the temperature at the Normal electrode. On the other hand, the rate of unwanted intra-gap events increases when $V_{S D}$ approaches $2 \Delta$, as described by eq. (19). Thus, the probability of both kinds of spurious events described by eqs. (19) and (20) reaches a maximum value either for $\mathrm{V}_{\mathrm{SD}}=2 \Delta$ or $\mathrm{V}_{\mathrm{SD}}=0$, respectively. Minimizing the contributions displayed in eqs. (19) and (20) leads to $\mathrm{V}_{\mathrm{SD}}=\Delta$.

It could seem, at a first sight, that the incorporation of superconductors with larger $\Delta$ is, at first sight advisable, if a drastic suppression of thermal error rates is required as in the case of metrological applications. This because larger $\Delta$ values would in principle allow operating the device at higher $\mathrm{V}_{\mathrm{SD}}$ bias.

Examples of h-SETs in literature generally employ $\mathrm{Al}$ as the superconductive component $(\Delta \approx 170 \mu \mathrm{eV})$. Apart from the ease of producing efficient dielectric junction barriers by means of simple $\mathrm{Al}$ oxidation, the $\Delta$ value for $\mathrm{Al}$ is relatively low, if compared e.g with $\mathrm{Nb}$ $(\Delta \approx 1.4 \mathrm{meV})$. As a matter of fact, there are limitations in employing larger gap superconductors $(\mathrm{Pb}, \mathrm{Nb})$ in state-of-art hybrid SETs. Such limitations are either of fundamental or of technological nature. In the followings we will discuss both these aspects.

\subsubsection{A scaling rule}

The capability of a h-SET device to act as a single elecron turnstile is related to the possibility of switching the system between two stable states A and B (Fig. 5), keeping the system in a blocked region of the stability diagram. All paths at nonzero $V_{S D}$ values which connect A and B, necessarly contain a set of states where the current is suppressed by means of the superconducting gap, solely. In the present chapter we consider the simplest theoretical and experimental setup for a turnstile with dc bias and ac gate voltage: in this framework the system switches between two blocked states, the first related to the Coulomb blockade in analogy with the n-SET and depicted by means of the AA' and BB' segments, the second represented by the $\mathrm{A}^{\prime} \mathrm{B}^{\prime}$ segment in which the tunnel current is suppressed by the superconducting gap. As previously discussed the superconductive gap cannot be considered as a perfect barrier and the transition in the $\mathrm{A}^{\prime} \mathrm{B}^{\prime}$ segment is a potential source of current leakage inside the tunnel junctions, then some considerations are needeed in order to minimize this effect mantaining the advantages of h-SET turnstile configuration.

Minimizing the resident time $t_{\Delta}$ in this region is then an important issue in order to reduce errors related to leakage effects. Authors (Pekola et al., 2008), suggested a squared waveform for the $\mathrm{V}_{\mathrm{g}}$ signal, even if the sinusoidal signal can be more easily handled during a turnstile experiment.

Evaluation of such resident time is easily obtained in the case of sinusoidal waveform, by considering the extension of the $\mathrm{A}^{\prime} \mathrm{B}^{\prime}$ region in Fig. 5 . We consider a value for $\mathrm{V}_{\mathrm{SD}}=\Delta$ (with $\Delta$ in $\mathrm{eV}$ ), say, we assume the SET as working in the optimal conditions according to eqs. (19) and (20). From geometrical considerations, as can be evident when observing Fig. 5, the condition:

$$
\Delta<\mathrm{E}_{\mathrm{c}}
$$



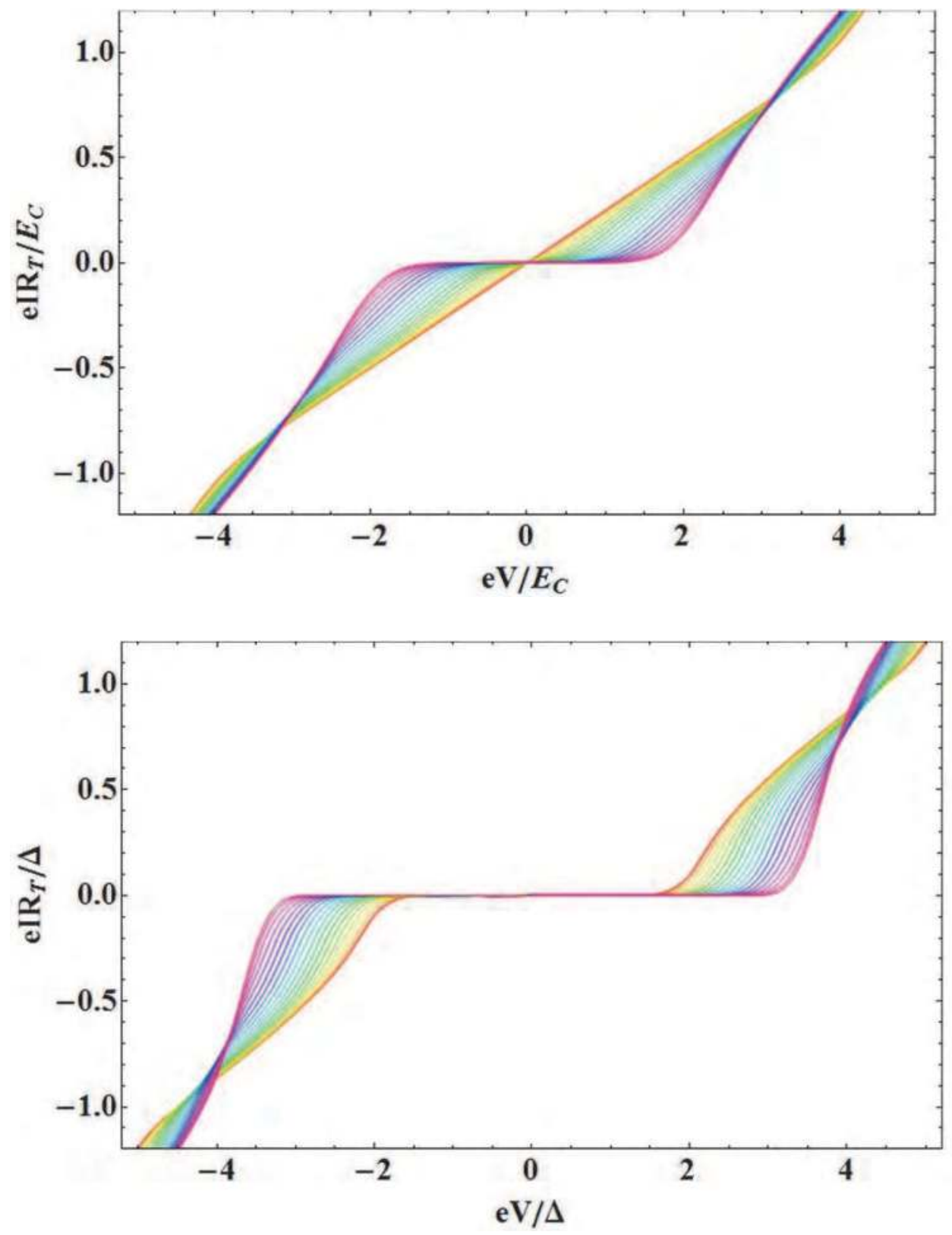

Fig. 6. Comparison between the Current-Voltage characteristics of a Normal (top) and hybrid (bottom) SET, taken at different gate voltage values. According to the Stability Diagram of Fig. 2 we observe the broadening of the Coulomb gap with varying $V_{g}$. In the hybrid assembly the contribution from $\Delta$ broadens the region of inhibited tunneling. 
must hold, otherwise the system will never reach a stable Coulomb-blocked state. Such simple relationship provides an important scaling rule for designing h-SETs. It says that employing high gap superconductors ( $\mathrm{Nb}$ is a key example) into a hybrid assembly does not guarantee better device performances. That is, the Charging Energy $\mathrm{E}_{\mathrm{C}}$ must be increased, too. As an example, if we envisage to replace $\mathrm{Al}$ with $\mathrm{Nb}(2 \Delta \approx 340 \mu \mathrm{eV}$ vs. $2 \Delta \approx 3 \mathrm{meV})$, we have to find a way to increase the $\mathrm{E}_{\mathrm{C}}$ value by a factor of $\sim 10$; this can be accomplished by decreasing the tunnel capacitance values, solely.

The ratio between $t_{\Delta}$ the time interval in which the system is blocked only by the superconductive gap during a cycle, and the cycle half-period $\mathrm{T} / 2$, can be written as:

$$
2 t_{\Delta} / T=\frac{1}{\pi}\left[\operatorname{arcos}\left(-\Delta / E_{c}\right)-\operatorname{arcos}\left(\Delta / E_{c}\right)\right]
$$

and displayed as a function of the junction capacitance and the superconducting gap $\Delta$ (Fig. 7). The $2 t_{\Delta} / \mathrm{T}$ ratio is $<1$ (indicative for the presence of a Coulomb Blockade region in the Stability Diagram, see Fig. 5) only in the portion of the $\Delta$-C plane in which the values of $\Delta$, and/or $\mathrm{C}$ are low. As a comparison, $\Delta$-values for typical low- $\mathrm{T}_{\mathrm{c}}$ superconductors are indicated together with the reasonnable lower limits for junction capacitance with the most common SET technologies, the SAIL (Self Aligning In-Line) (Götz et al., 1996) and the Shadow evaporation (Dolan, 1977).

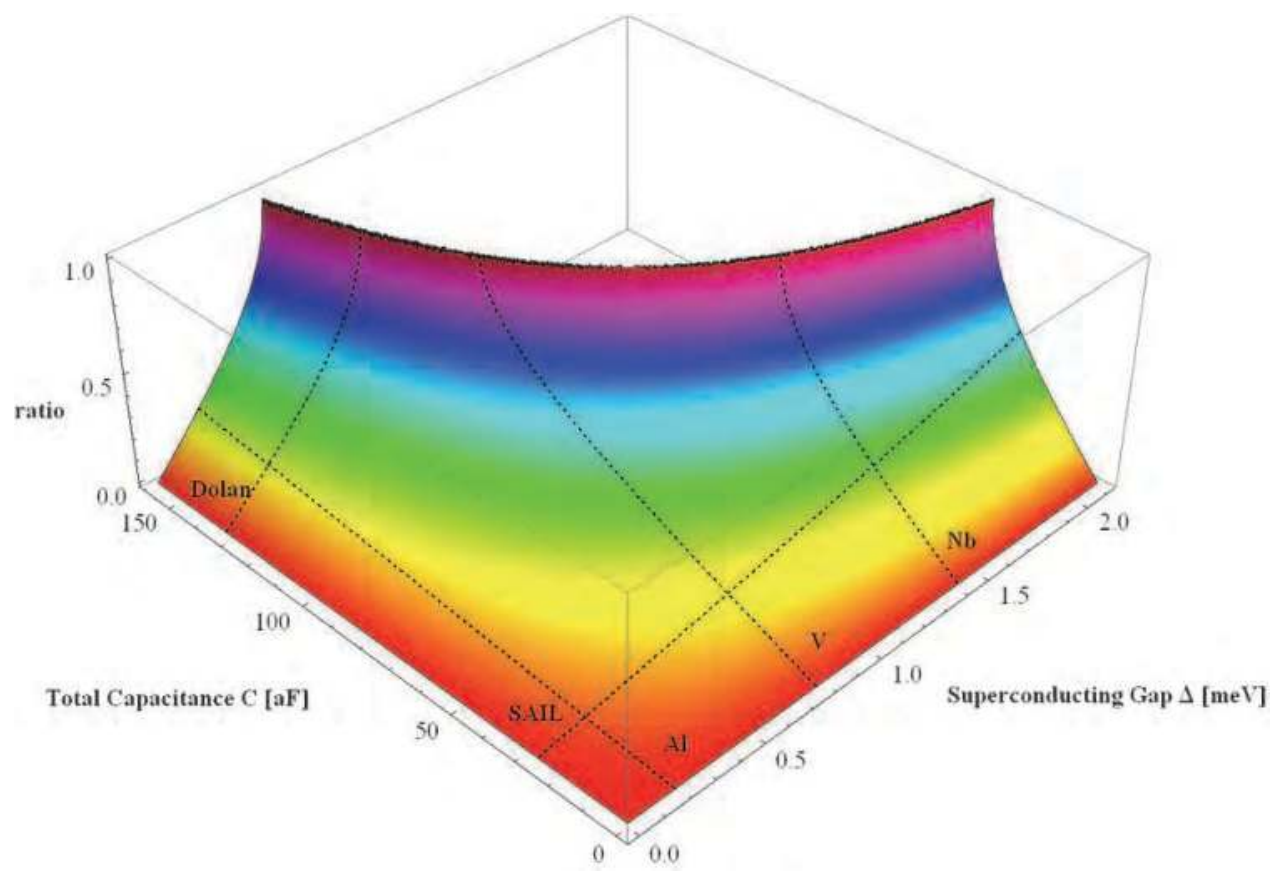

Fig. 7. The graph displays the calculated dependence of The $2 \mathrm{t} \Delta / \mathrm{T}$ on the superconductor gap $\Delta$ and the junction capacitance $C$. Lines perpendicular to the $\Delta$-axis show the typical gap values for most common low- $\mathrm{T}_{\mathrm{c}}$ superconductors, whereas the lines across the $\mathrm{C}$-axis represent the limit of two typical techniques for producing SETs (see text for details). 
The following chapter will review the technological approaches to realize SET devices, with the purpose of identifying the most promising ones as far as the capacitance reduction issue is concerned.

\section{SET Technologies}

\subsection{The Shadow evaporation technique}

The shadow evaporation technique (Dolan, 1977) was the first to be used for the fabrication of single-electron devices based on metallic systems and is currently the most widespread. This technology takes advantage from a shadow effect, implying that the deposition techniques must be highly non-conformal. The typical deposition process is then thermal or, better, e-beam evaporation: this dramatically limits the choice of materials to be deposited $(\mathrm{Nb}$, for example, being a refractory material, is hardly evaporated).

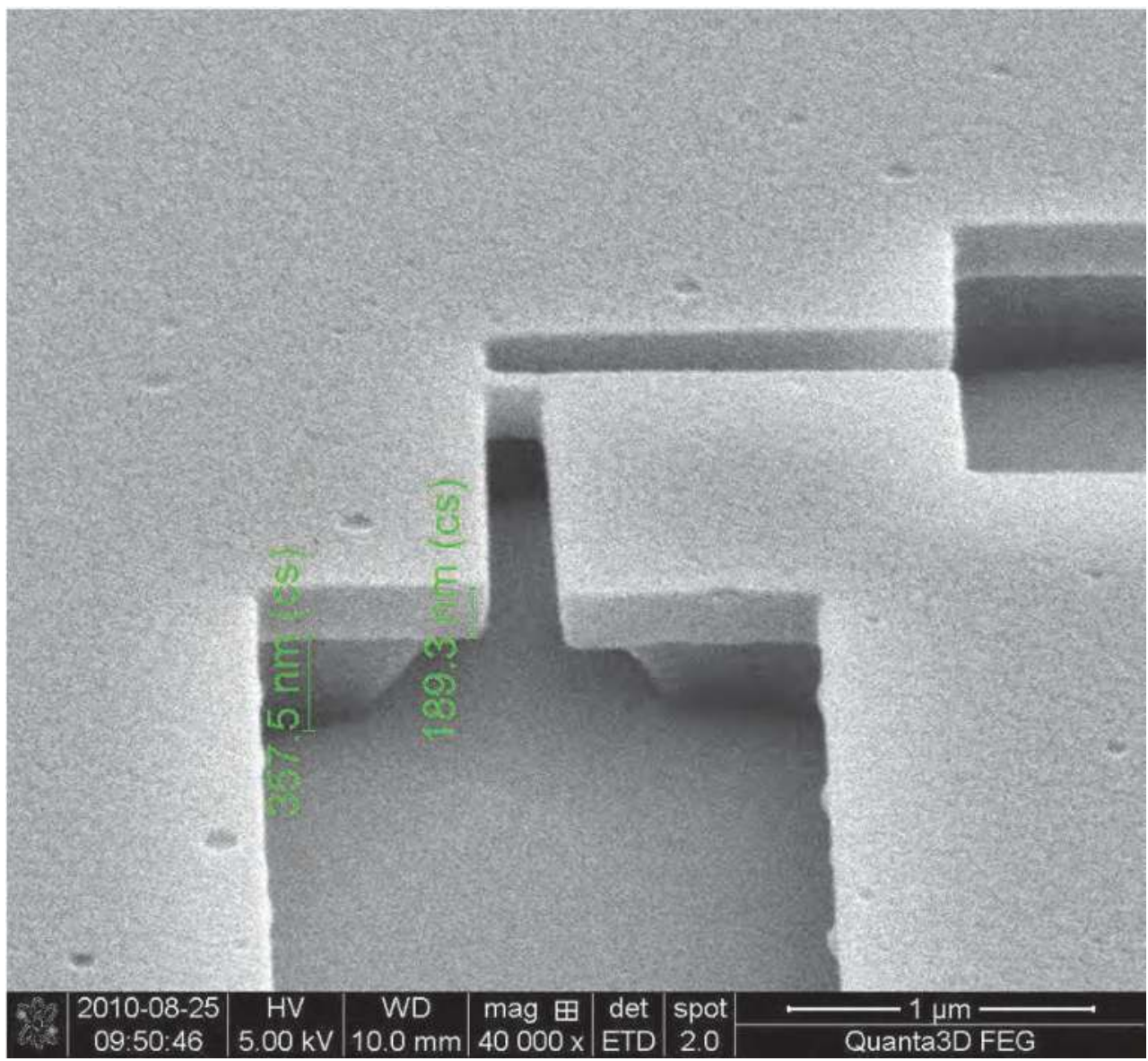

Fig. 8. SEM image of a suspended mask for Shadow evaporation. 
The critical step for the success of the process is to fabricate suspended segments of electron beam resist at a certain distance from the substrate. In common lift-off process, the films are defined by evaporating the metal through the openings in the mask at normal incidence substrate, so as to ensure the break between the parts of the layer on the substrate and those on the mask.

The creation of masks with suspended bridges is possible thanks to the use of two different types of resists for electron beam lithography, the lower with greater sensitivity to electron beam than the upper one. During the development step, the exposed resist region is chemically removed in a selective way, with a wider pattern in the polymer underneath. In this way, using the so-called proximity effect, typical of electron beam lithography, it is possible to obtain suspended bridges structures.

Fig. 8 shows the SEM tilted view of the mask we are dealing with: it consists of a support resist layer of thickness $\delta_{1} \sim 350 \mathrm{~nm}$, on which the layer that define the structures, with thickness $\delta_{2} \sim 200 \mathrm{~nm}$ is lying.

If the mask is suspended one no longer needs to deposit the metal at normal incidence to guarantee the successful lift-off and can vary the angle of deposition thus obtaining different patterns on the substrate. From simple geometrical considerations we can see that creating an opening of width $W_{0}$ in the top layer of resist and carrying out the evaporation at an angle $\Theta$ respect to the normal will produce a deposided feature of width:

$$
W_{0}=\delta_{2} \tan (\Theta)
$$

If the angle of incidence is greater than the critical one:

$$
\Theta>\Theta_{0}=\arctan \left(W_{0} / \delta_{2}\right)
$$

the opening in the mask appears as "closed" and the deposition does not reach the substrate.

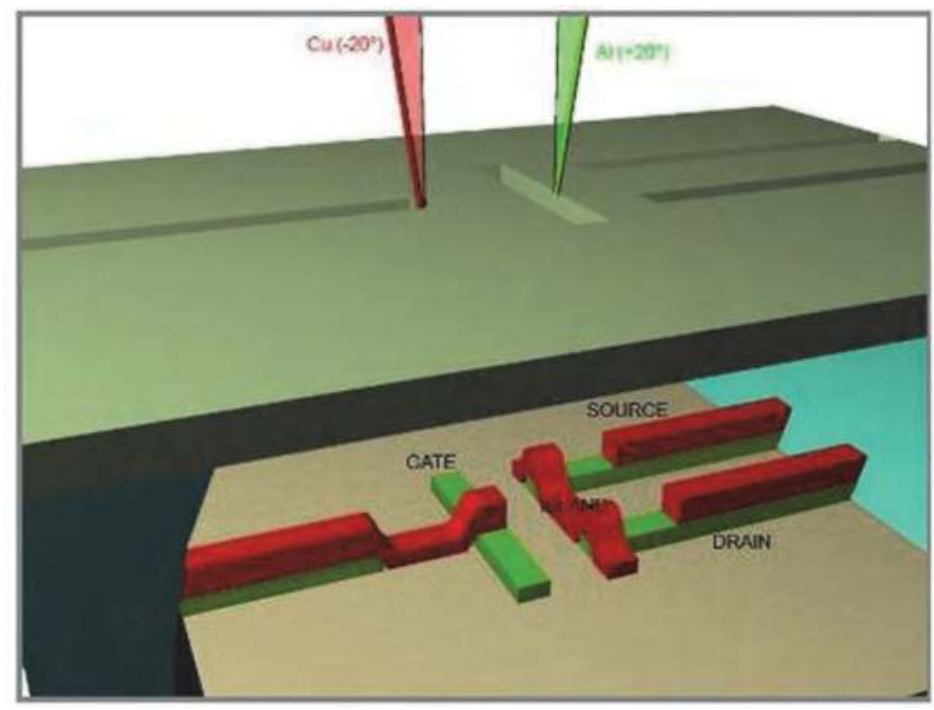

Fig. 9. Schematics of the angled Shadow evaporation process 
The practical realization of this effect depends on the ability to produce shadow masks similar to the ideal ones presented so far. To apply this calculation it is important that the experimental values of $\delta_{1}$ and $\delta_{2}$ are reliable, and that the cross section of the top resist layer is rectangular.

For the construction of tunnel junctions, first a pattern mask with two, very tight openings must be created. A bridge in the top layer resist between them is then defined.

One can then proceed to the fabrication of tunnel junctions with a deposition-oxidationdeposition sequence, which occurs in the same vacuum cycle. After the first evaporation performed to an angle $\delta_{1}$, (Al in Fig. 9) the deposited film is oxidized in $\mathrm{O}_{2}$ atmosphere then growing an insulating layer, commonly $\mathrm{Al}$ oxide, $1 \mathrm{~nm}$ thick. After pumping down, the second layer is then deposited at angle $\delta_{2}$ (Cu in Fig. 9).

\subsection{The Self Aligning In Line Process (SAIL)}

The principle of the SAIL technique (Koch, 1987) is to fabricate the tunnel junctions at the two sides of the island, so that the size of the junctions is determined by the thickness and width of metal thin films: in this way one gets a planar configuration with vertical barriers. In this section we will discuss the basic steps of the process originally created and provide some hints on how it could be used for manufacturing h-SETs.

The SAIL process, as presented by Gotz (Gotz et al., 1995) consists of the following steps:

i. Preparation of a narrow and thin metal film on the substrate (Fig. 10 (a)).

ii. Fabrication of a resist mask which leaves the area open for the following counter electrode deposition step (Fig. 10 (b)).

iii. Anisotropic etching of the film in order to define the island (Fig. 10 (c)).

iv. Formation of a dielectric barrier on the exposed surface of the island (Fig. 10 (d))

v. Deposition of the second metal film (Fig. 10 (e)).

vi. Lift off (Fig. 10 (f)).

There are no particular requirements for the island deposition technique, e.g. sputtering or evaporation, while the subsequent transfer of the pattern can be accomplished with lift off or anisotropic etching.

The mask generated in the second step defines the location and size of the island and that of source and drain electrodes. The process is self-aligned along the length of the island, while mismatches in the cross direction can be easily compensated by choosing one of the two metal strips wider: then one can realize an island sandwiched with two wide electrodes (WNW), as shown in Figure 9, or a large island between two narrow electrodes (NWN), obtaining in both cases the same junction area.

Difficulties could arise from the use of the same mask for etching and lift off: in fact, the resist must remain soluble and thick enough to allow reliable lift off, even after the ion beam bombardment. One will then need to tune the thickness of the resist or the metal depending on the etching selectivity. The solution may be to replace the ion beam etching, barely physical, with Reactive Ion Etching (RIE), taking advantage from the chemical selectivity of the gas employed.

An alternate solution is the use of a multi-layered mask, e.g. two layers of resist with an intermediate layer with lower etching rate. In this way, the lower resist layer is protected against the ion bombardment, and can be used as lift off mask. 

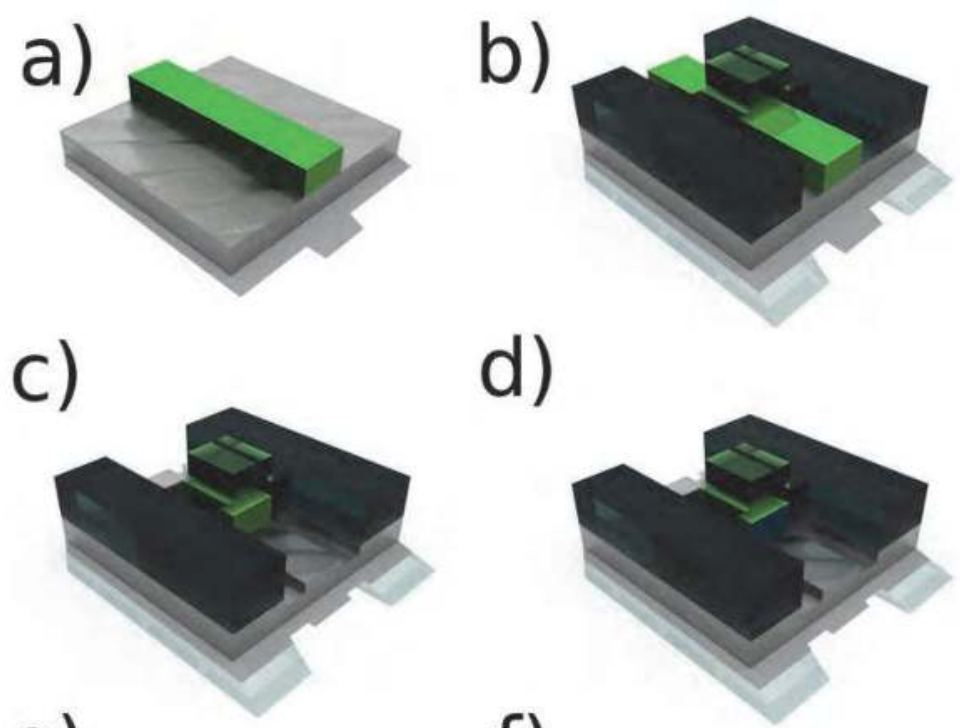

d)

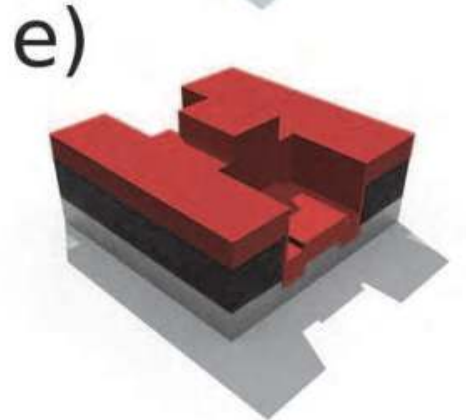

f)
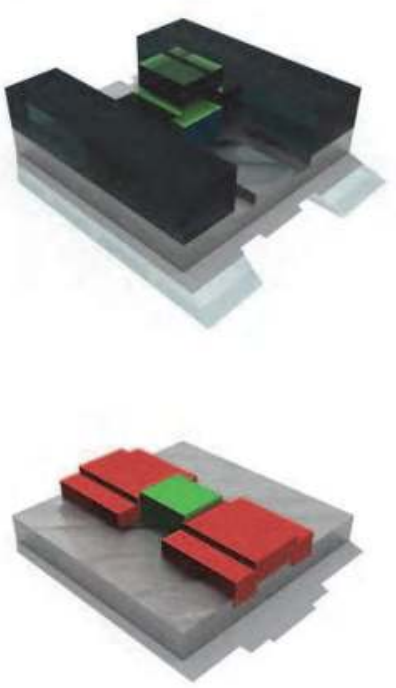

Fig. 10. The main technological steps for the SAIL technique. See text for details.

In order to be used for lift off, the resist mask should show in section walls with negative slope. The generation of a suitable mask is the crucial step and more complicated in the SAIL technique than in the shadow evaporation one.

The creation of the barrier after the anisotropic etching of the first mask avoids its damage due to high-energy ions.

Over-etching in the substrate during step iii. can lead to re-deposition of substrate material on the exposed sides of the island, and then serious barrier uniformity problems can arise. To improve the quality of the barrier as well as to minimize the over-etching, it is possible to choose as substrate the same material of the barrier to be fabricated: in fact, the barrier dielectrics usually have lower etching rates than the corresponding pure metals, and therefore can excellently act as etch-stop layers.

A further technological complication is that the formation of reliable contacts requires a more anisotropic etching (step iii.) than the second metal deposition step (step vi.).

Apart from these difficulties, the SAIL process has several advantages if compared to the shadow evaporation technique. 
As mentioned above, there is complete freedom in the choice of the deposition process of metal layer, e.g. evaporation can be replaced by sputtering. It is worth noting, for instance, that the latter technique is more suitable for depositing a robust and reliable superconductor like $\mathrm{Nb}$. Moreover, one can get rid of fragile structures like suspended bridges necessary for the shadow evaporation. Finally, since the tunnel junction is obtained at the sides of the island, the electrodes overlapping is absent, and the junction capacitance is lower than in devices realized by the shadow evaporation.

The first SET made with the SAIL technique was reported by M. Gotz (Gotz et al., 1995). The device is based on the system $\mathrm{Al} / \mathrm{AlO}_{x} / \mathrm{Al}$. The island, with thickness and width of $50 \mathrm{~nm}$ and $80-150 \mathrm{~nm}$, respectively, is defined by EBL and subsequent lift off on a single layer of AR-P610 resist. The metal was deposited by sputtering. The second mask was made with a double resist layer composed by AR-P671 and AR-P 641. The thickness of the second metal layer was $100 \mathrm{~nm}$. The anisotropic etching was carried out with $\mathrm{Ar}^{+}$ions. Immediately after the etching, the dielectric barrier has been created by means of oxidation step in dry air. The reported yield is $40 \%$.

From the width of the Coulomb Blockade areas, the junction capacitance was estimated to be $0.5 \mathrm{fF}$, a value in agreement with the calculations for a tunnel junction area of $50 \times 150$ $\mathrm{nm}^{2}$, and a barrier thickness of the order of $1 \mathrm{~nm}$.

\section{Conclusions}

The employment of the Shadow evaporation technique dramatically limits the choice for superconductors to use, either from a merely technical (materials to be evaporated) or from a more fundamental (difficulties in reducing junction areas) points of view. As a matter of fact, h-SETs made by $\mathrm{Al} / \mathrm{Cu}$ assemblies have been recently produced and characterized (Pekola et al., 2008). The SAIL technique seems promising, since it allows for a wider choice of superconducting materials. It is possible, for example, to envisage the employment of $\mathrm{In}: \mathrm{Pb}$ alloys (with improved electrical and thermal properties with respect to the unalloyed elements) in SAIL SETs by taking advantage from composition-related gap tunability. In this case, however, technological problems related to deposition of continuous, $\sim 10 \mathrm{~nm}$ thick, films from metals with low fusion temperature require solution. It is noteworthy that such alloys were used years ago in the first generation Josephson junctions (Lacquaniti et al., 1982). V or Ta could be interesting alternatives, but the best candidate for the realization of stable and robust turnstiles should obviously be $\mathrm{Nb}$. Indeed, the graph in Fig. 7 shows that the inclusion of such material in a h-SET arrangement still requires to overcome the technological limitations of the SAIL technique.

The possibility of device biasing, offered by the hybrid arrangement can improve the accuracy of electron pumping process, but care must be taken in reducing leakage through the superconducting gap. Optimizing between these opposite effects requires the increase of both the superconducting gap and the charging energy.

\section{Acknowlegdments}

The work has been carried out at Nanofacility Piemonte supported by Compagnia di San Paolo. 


\section{References}

Altshuler, B. L.; Lee, P. A. \& Webb, R. A. (1991). Mesoscopic phenomena in solids. North Holland, 1991, 978-044-4884-54-1

Averin, D. V.; Korotkov, A. N. \& Likharev, K. K. (1991). “Theory of single-electron charging of quantum wells and dots," Physical Review B, vol. 44, n. 12, pag. 6199, 1991.

Averin, D. V. \& Pekola, J. P. (2008). “Nonadiabatic Charge Pumping in a Hybrid SingleElectron Transistor," Physical Review Letters, vol. 101, n. 6, pag. 066801, 2008.

Blumenthal, M. D.;Kaestner, B.;Li, L.;Gibling, S.;Janssen, T.J.B.M.;Pepper, M.;Anderson, D.jones, G. \& Ritchie, D.A. “Gigahertz quantized charge pumping," Nat Phys, vol. $3, n^{\circ} .5,(2007)$

Cholascinski, M. \& Chhajlany, R. W. (2007). “Stabilized Parametric Cooper-Pair Pumping in a Linear Array of Coupled Josephson Junctions," Physical Review Letters, vol. 98, n. 12, pag. 127001, Mar. 2007.

Clark, A. M. (2005).;Miller, N.A.;Williams, A.;Ruggiero, S.T.;Hilton, G.C.;Vale, L.R.;Beall, J.A.; Irwin, K.D. \& Ullom, J.N. , “Cooling of bulk material by electron tunneling refrigerator ". Appl. Phys. Lett. 86, 173508 (2005).

Dolan, G. J. “Offset masks for lift-off photoprocessing”. Appl. Phys. Lett. 31, 337 (1977).

Flowers, J. (2004). "The Route to Atomic and Quantum Standards," Science, vol. 306, n . 5700, pagg. 1324-1330, Nov. 2004.

Geerligs, L. J. ; Anderegg, V. F.; van der Jeugd, C. A.; Romijn, J. \& Mooij, J. E. (1989). "Influence of Dissipation on the Coulomb Blockade in Small Tunnel Junctions" Europhys. Lett. 10, 79, 1989.

Geerligs, L. J.; Anderegg, V.F.;Holweg, P.A.M.; Mooij, J.E.; Poithier, H.; Esteve, D.; Urbina, C. \& Devoret, M.H. (1990) "Frequency-locked turnstile device for single electrons," Physical Review Letters, vol. 64, n. 22, pag. 2691, Mag. 1990.

Giazotto, F.; Heikkilä, T. T.; Luukanen, A.; Savin, A. M. \& Pekola, J. P. 2006). “Opportunities for mesoscopics in thermometry and refrigeration: Physics and applications" Rev. Mod. Phys. 78, 217 (2006).

Governale, M.; Taddei, F.; Fazio, R. \& Hekking, F. W. J. (2005). “Adiabatic Pumping in a Superconductor-Normal-Superconductor Weak Link," Physical Review Letters, vol. 95, n. 25, pag. 256801, Dic. 2005.

Götz, M.; Bluthner, K.; Krech, W.,Nowack, A.; Fuchs, H.J.; Kley, E.B.; Thieme, P.; Wagner, Th. Eska, G.; Hecker, K. \& Hegger, H. (1996) "Self-aligned in-line tunnel junctions for single-charge electronics," Physica B: Condensed Matter, vol. 218, n. 1, pagg. 272275, Feb. 1996.

Ingold, G. L. \& Nazarov, Y. V. (1992) in Single charge tunneling, Vol. 294 of NATO ASI Series B, edited by H. Grabert \& M. H. Devoret, Plenum Press, New York, 1992, ISBN 0-306-44229-9.

Josephson, B. D. (1962). "Possible new effects in superconductive tunneling," Physics Letters, vol. 1, n. 7, pagg. 251-253, Lug. 1962.

Keller, M. W.; Martinis, J. M.; Zimmerman, N. M. Steinbach, \& A. H. (1996). “Accuracy of electron counting using a 7-junction electron pump," Applied Physics Letters, vol. 69, $\mathrm{n}^{\circ} .12$, pag. 1804, 1996. 
Koch H. "Self-Aligned In-Line Junction - Fabrication and Application to DC-SQUIDS", Int. Supercond. Electr. Conf., Tokyo August 1987 (Extended Abstracts) pp. 281-284.

Kopnin, N. B.; Mel'nikov, A. S. \& Vinokur, V. M. (2006). "Resonance Energy and Charge Pumping through Quantum SINIS Contacts," Physical Review Letters, vol. 96, n². 14, pag. 146802, Apr. 2006.

Koppinen, P.; Kühn, T. \& Maasilta, I. (2009), “Effects of Charging Energy on SINIS Tunnel Junction Thermometry “ J. Low Temp. Phys. 154, 179 (2009).

Lacquaniti, V; Battistoni, C.; Paparazzo, E.; Cocito, M.; Palumbo, S. (1982). “Tunnelling barrier structure of $\mathrm{Nb} / \mathrm{Pb}$ and $\mathrm{Nb} /(\mathrm{Pb}-\mathrm{In})$ thin film Josephson junctions studied by Auger electron spectroscopy and x-ray photoelectron spectroscopy analysis, " Thin Sol. Films, vol. 94, ${ }^{\circ}$. 4, pagg. 331-339. 1982

Likharev, K. K. (1988) "Correlated discrete transfer of single electrons in ultrasmall tunnel junctions," IBM Journal of Research and Development, 1988.

Lotkhov, S. V. (2004)"Radio-frequency-induced transport of Cooper pairs in superconducting single electron transistors in a dissipative environment," Journal of Applied Physics, vol. 95, n. 11, pag. 6325, 2004.

Meschke, M.; Guichard, W. \& Pekola, J. P. (2006). “Single-mode heat conduction by photons" Nature 444, 187 (2006).

Mooij, J. E. \& Nazarov, Y. V. (2006). “Superconducting nanowires as quantum phase-slip junctions," Nature Physics, vol. 2, nº. 3, pagg. 169-172, Mar. 2006.

Nahum, M.; Eiles, T. M. \& Martinis, J. M. (1994). “Electronic microrefrigerator based on a normal-insulator-superconductor tunnel junction“ Appl. Phys. Lett. 65, 3123 (1994).

Nahum, M.; \& Martinis, J. M. (1993). “Ultrasensitive-hot-electron microbolometer" Appl. Phys. Lett. 63, 3075 (1993).

Niskanen, A. O.; Pekola, J. P. \& Seppä, H. (2003). “Fast and Accurate Single-Island Charge Pump: Implementation of a Cooper Pair Pump," Physical Review Letters, vol. 91, $\mathrm{n}^{\circ}$. 17, pag. 177003, Ott. 2003.

Pekola, J. P.; Giazotto, F. \& Saira, O.-P. (2007). "Radio-Frequency Single-Electron Refrigerator" Phys. Rev. Lett. 98, 037201 (2007).

Pekola, J. P.; Vartiainen, J. J.; Mottonen, M.; Saira, O.-P.; Meschke, M. \& Averin, D. V. (2008). "Hybrid single-electron transistor as a source of quantized electric current," Nat Phys, vol. 4, $\mathrm{n}^{\circ}$. 2, pagg. 120-124, 2008.

Pothier, H.; Lafarge, P.; Urbina, C.; Esteve, D. \& Devoret, M. H. (1992). "Single-Electron Pump Based on Charging Effects," Europhysics Letters (EPL), vol. 17, n. 3, pagg. 249-254, 1992.

Saira, O.-P.; Meschke, M.; Giazotto, F.; Savin, A. M.; Möttönen, M. \& Pekola, J. P. (2007). Phys. Rev. Lett. 99, 027203 (2007).

Schmidt, D. R.; Yung, C. S. \& Cleland, A. N. (2003). “Nanoscale radio-frequency thermometry“ Appl. Phys. Lett. 83, 1002 (2003).

Talyanskii, V. I.; Shilton, J.M.; Pepper, M.; Smith, C.G.; Ford, C.J.B.; Linfield, E.H.; Ritchie, D.A. \& Jones, G.A.C. (1997). "Single-electron transport in a one-dimensional channel by high-frequency surface acoustic waves," Physical Review B, vol. 56, nº. 23, pag. 15180, Dic. 1997. 
Vartiainen, J. J.; Möttönen, M.; Pekola, J. P. \& Kemppinen, A. (2007). “Nanoampere pumping of Cooper pairs," Applied Physics Letters, vol. 90, n. 8, pag. 082102, 2007.

Zimmerman, N. M. \& Keller, M. W. (2003). "Electrical metrology with single electrons," Measurement Science and Technology, vol. 14, n. 8, pagg. 1237-1242, 2003.

Zwerger, W. \& Scharpf, M. (1991). “Crossover from Coulomb-blockade to ohmic conduction in small tunnel junctions," Zeitschrift für Physik B Condensed Matter, vol. 85, n 3. 


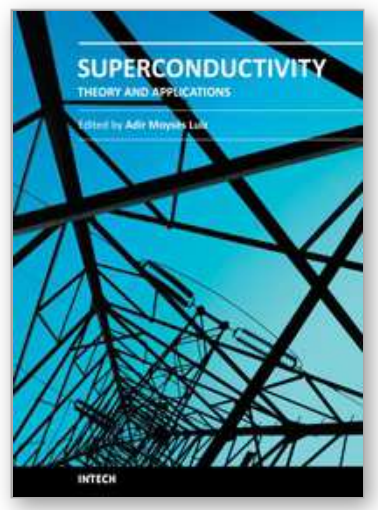

\section{Superconductivity - Theory and Applications}

Edited by Dr. Adir Luiz

ISBN 978-953-307-151-0

Hard cover, 346 pages

Publisher InTech

Published online 18, July, 2011

Published in print edition July, 2011

Superconductivity was discovered in 1911 by Kamerlingh Onnes. Since the discovery of an oxide superconductor with critical temperature (Tc) approximately equal to $35 \mathrm{~K}$ (by Bednorz and Müller 1986), there are a great number of laboratories all over the world involved in research of superconductors with high Tc values, the so-called â€œHigh-Tc superconductorsâ€. This book contains 15 chapters reporting about interesting research about theoretical and experimental aspects of superconductivity. You will find here a great number of works about theories and properties of High-Tc superconductors (materials with Tc $>30 \mathrm{~K}$ ). In a few chapters there are also discussions concerning low-Tc superconductors ( $\mathrm{Tc}<30 \mathrm{~K}$ ). This book will certainly encourage further experimental and theoretical research in new theories and new superconducting materials.

\section{How to reference}

In order to correctly reference this scholarly work, feel free to copy and paste the following:

Giampiero Amato and Emanuele Enrico (2011). Current Status and Technological Limitations of Hybrid Superconducting-Normal Single Electron Transistors, Superconductivity - Theory and Applications, Dr. Adir Luiz (Ed.), ISBN: 978-953-307-151-0, InTech, Available from:

http://www.intechopen.com/books/superconductivity-theory-and-applications/current-status-and-technologicallimitations-of-hybrid-superconducting-normal-single-electron-transi

\section{INTECH}

open science | open minds

\section{InTech Europe}

University Campus STeP Ri

Slavka Krautzeka 83/A

51000 Rijeka, Croatia

Phone: +385 (51) 770447

Fax: +385 (51) 686166

www.intechopen.com

\section{InTech China}

Unit 405, Office Block, Hotel Equatorial Shanghai

No.65, Yan An Road (West), Shanghai, 200040, China

中国上海市延安西路65号上海国际贵都大饭店办公楼 405 单元

Phone: +86-21-62489820

Fax: $+86-21-62489821$ 
(C) 2011 The Author(s). Licensee IntechOpen. This chapter is distributed under the terms of the Creative Commons Attribution-NonCommercialShareAlike-3.0 License, which permits use, distribution and reproduction for non-commercial purposes, provided the original is properly cited and derivative works building on this content are distributed under the same license. 